\title{
Compétence en communication professionnelle en santé
}

\section{Professional competence in healthcare}

\section{Claude RICHARD ${ }^{1}$, Marie-Thérèse LuSSIER ${ }^{1,2}$, Sophie GALARNEAU ${ }^{2,3}$ et Olivier JAMOULLE ${ }^{4,5}$}

1 Équipe de recherche en soins de première ligne, Cité de la santé de Laval, Laval, Canada

2 Département de médecine familiale et de médecine d'urgence, Faculté de médecine, Université de Montréal, Montréal, Canada

3 Centre de santé et de services sociaux Jeanne-Mance/Centre local de services communautaires des Faubourgs, Montréal, Canada

4 Département de pédiatrie, CHU Sainte Justine, Université de Montréal, Montréal, Canada

5 CHU Sainte Justine, Montréal, Canada

Manuscrit reçu le 4 mars 2010 ; commentaires éditoriaux formulés aux auteurs le 9 octobre 2010 ; accepté pour publication le $1^{\text {er }}$ février 2011

\section{Mots clés :}

Compétence ;

communication

professionnelle ;

communication

médecin-patient ;

relation médecin-patient

\section{Keywords:}

Competence;

professional

communication;
Résumé - Contexte : Les initiatives pédagogiques en enseignement de la communication restent trop souvent théoriques et cloisonnées. Dans le cadre d'un projet pédagogique facultaire orienté sur l'approche par compétences, un groupe de travail a recensé les contenus de la formation en communication médicale à donner et a identifié les habiletés communicationnelles à maîtriser. La description des différents contenus et habiletés en communication professionnelle en médecine permettra de guider les étudiants dans leur curriculum de formation et de soutenir les enseignants dans le développement d'activités pédagogiques. Méthodes : Depuis 2007, un groupe de représentants des départements de médecine familiale, de médecine d'urgence, d'autres spécialités médicales et chirurgicales, d'experts en pédagogie et en communication et un étudiant s'est réuni régulièrement. Il a proposé une définition et une description de l'ensemble des constituantes de la communication médicale en tenant compte des données scientifiques publiées en communication et en pédagogie. Résultats : Une proposition d'organisation des connaissances et des habiletés nécessaires au développement de la compétence en communication a émergé du travail du « conseil de compétence communication ». La représentation développée suggère une approche systématique et graduelle de la formation où la réussite repose sur la maîtrise progressive des différentes constituantes. Ainsi, les formateurs disposeront d'un contexte théorique, d'une identification des différents savoirs et savoir-faire nécessaires à une communication professionnelle efficace, ainsi que d'un outil pédagogique sous forme d'une roulette combinant les différentes facettes de la communication et les multiples situations professionnelles que le praticien rencontre. Conclusion : Ce document servira de base aux responsables pédagogiques facultaires et aux enseignants pour organiser l'enseignement de la communication dans leur spécialité.

Abstract - Introduction: It is now widely accepted that medical communication training should be rooted in clinical practice. Still, many pedagogical initiatives remain theoretical and cut off from clinical activities. Moreover, these initiatives seldom consider the most appropriate and proven effective teaching methods. The objective of the University of Montreal 


$\begin{array}{ll}\text { doctor-patient } & \text { faculty of medicine working group was to define the content of medical communication train- } \\ \text { communication; } & \text { ing and determine the necessary communication skills to be mastered and expected compe- } \\ \text { doctor-patient } & \text { tencies for each level of medical education. Methods: A group of medical educators from } \\ \text { relationship } & \text { departments of family medicine, medical and surgical specialties, as well as pedagogy and } \\ \text { communication experts and a student representative have met regularly since 2007. Together } \\ \text { they developed a framework of medical communication. To ensure coherence throughout the } \\ \text { medical curriculum, the committee members based their work on published research in the } \\ \text { fields of communication as well as pedagogy. Results: The committee produced a framework } \\ \text { of the necessary knowledge and skills necessary to acquire competence in medical communi- } \\ \text { cation. The resulting representation suggests a systematic and progressive approach to com- } \\ \text { munication training. Thus, medical trainers now have a theoretical framework as well as a } \\ \text { training tool in the form of a competence wheel that allows them to combine different com- } \\ \text { munication elements relevant to diverse clinical situations that physicians encounter in their } \\ \text { practice. Discussion: This document will serve as the basis on which medical educators and } \\ \text { teachers can organize communication training with respect to their specialty. }\end{array}$

\section{Introduction}

Dans le cadre d'un projet pédagogique facultaire basé sur une approche par compétences, la faculté de médecine de l'Université de Montréal a mis sur pied plusieurs groupes de travail, connus sous le nom de « conseils de compétence ». Le mandat de notre groupe consistait à définir le contenu de la formation en communication médicale, à déterminer les habiletés à maîtriser et à identifier les compétences attendues.

Nous croyons que le travail du conseil de compétence en communication, bien que toujours en cours, a atteint un degré de maturité suffisant qui permet de le partager avec l'ensemble de la communauté francophone de pédagogie en sciences de la santé.

Dans le présent article, nous proposons une organisation explicite et systématique de différents concepts et situations liés à la communication professionnelle en médecine. Cette proposition a l'ambition de constituer un référentiel didactique pour construire des programmes ou des curriculums dédiés et ne constitue pas, dans sa forme actuelle, un référentiel pédagogique. L'exploitation pédagogique qui peut en être faite n'est ici qu'esquissée. Les principes pédagogiques directeurs d'enseignement, d'apprentissage et d'évaluation restent à développer à partir de l'organisation de la communication professionnelle en médecine proposée ici.

\section{Problématique}

Il est maintenant admis que la maîtrise de la compétence de communication professionnelle en médecine doit reposer sur des assises scientifiques reconnues dans les domaines de la communication et de la pédagogie. L'apprentissage de cette compétence doit également être structuré de façon à permettre à l'étudiant son acquisition graduelle, intégrée à la pratique clinique et balisée tout au long d'un cursus longitudinal structuré.

Pourtant, même la définition de cette compétence ne fait pas encore l'unanimité et, jusqu'à présent, les connaissances reliées à ce champ de compétence professionnelle ont souvent été abordées de manière informelle et implicite au cours du curriculum des études médicales, sans référence à un cadre théorique rigoureux ${ }^{[1]}$. De plus, lorsque la communication est abordée, on semble privilégier des présentations magistrales plutôt que des approches interactives et expérientielles dont l'efficacité a été démontrée dans ce domaine ${ }^{[2]}$. Aussi, les modèles conceptuels existants de la compétence en communication médicale, tels que par exemple l'approche bio-psycho-sociale, l'approche centrée sur le patient ou l'approche centrée sur la relation, se focalisent le plus souvent sur les aspects de la relation clinique entre le médecin et le patient ${ }^{[3]}$. Plutôt que de porter leur attention à l'ensemble des occasions de 
communication offertes au médecin dans l'exercice de ses fonctions.

Le conseil de compétence en communication de l'Université de Montréal, riche de l'expertise et de l'expérience clinique et pédagogique de ses membres, s'est donné comme premier objectif de pallier les lacunes identifiées ci-dessus. Pour ce faire, les travaux du conseil se sont adossés aux principes directeurs issus de cadres conceptuels explicites reconnus dans le champ de la communication interpersonnelle ${ }^{[4-6]}$ et celui de la communication interpersonnelle appliquée à l'exercice de la profession médicale. En particulier les travaux du conseil se sont inspirés des modèles de von Fragstein et $a l .{ }^{[7]}$, de Silverman et $a{ }^{\left[{ }^{[8]}\right.}$ et de Lussier et Richard ${ }^{[9]}$ qui, tout en incorporant les valeurs implicites des modèles classiques mieux connus identifiés au paragraphe précédent, incluent les notions de contextes de la parole du médecin, du rôle des divers interlocuteurs avec lesquels le médecin doit interagir et des média de communication utilisés.

Dans le présent article, les auteurs, eux-mêmes membres du conseil de compétence, présentent le résultat des travaux du conseil compétence en communication. Ils proposent une définition élargie de la communication professionnelle qui englobe un ensemble de constituantes qui permettent de rendre compte de la complexité de la compétence en communication médicale. Ils proposent également une représentation graphique sous la forme d'une «roulette combinatoire » de cercles concentriques qui, en plus d'illustrer la pertinence de la communication en pratique clinique, la rend dynamique et applicable dans divers contextes et situations d'apprentissage tout au long de la formation médicale.

\section{Collectifs et méthode}

Les participants

Le comité central des compétences de la faculté de médecine de l'Université de Montréal a, dès 2007, mis en place sept conseils de compétence qui correspondaient au cadre de compétences CanMEDS 2005
Tableau I. Expertises des membres du conseil de compétence en communication (Faculté de médecine de l'Université de Montréal).

- Dr Joseph Ayoub, coprésident, oncologue

- Dr Olivier Jamoulle, coprésident, pédiatre

- Dr Andrée Boucher, endocrinologue, vice-doyenne

à la pédagogie et au développement professionnel continu, directrice du secteur Compétences du Centre de Pédagogie Appliquée en Sciences de la Santé (CPASS).

- Dr Josée Dubois, radiologue

- Dr Marie-Josée Dupuis, gynécologue-obstétricienne

- Dr Sophie Galarneau, médecin de famille

- Monsieur François Lagarde, expert-conseil en marketing social et en communications

- Dr Maude Lefebvre, médecin-résident en génétique

- Dr Marie-Thérèse Lussier, médecin de famille

- Dr Claude Richard, PhD psychologue, expert en communication

du Collège royal des médecins et des chirurgiens du Canada $^{[10]}$, à savoir l'expertise, la communication, la collaboration, le professionnalisme, l'érudition, la gestion et la promotion de la santé. Des membres des départements de médecine familiale et de médecine d'urgence, de gynéco-obstétrique, de radiologie, de médecine interne, d'oncologie, de pédiatrie ainsi que des experts en pédagogie et en communication et un représentant étudiant ont été invités à former le conseil de compétence en communication (tableau I).

Le processus de travail

Le conseil de compétence en communication $(n=9)$ s'est réuni sur une base régulière au cours des trois dernières années. Le groupe a utilisé l'ouvrage «La communication professionnelle en santé » ${ }^{[11]}$ comme outil de référence principal étant donné la participation des deux co-directeurs scientifiques du livre au conseil de compétence et la recension exhaustive des écrits sur la communication 
que ce volume contient. Cette information a été complétée par l'analyse d'autres articles scientifiques, de recherche et de formation continue portant sur la communication et son apprentissage. Des documents de travail ont été rédigés par les membres et présentés à l'ensemble du groupe qui les a commenté et amélioré. Les deux co-présidents du conseil ont participé à plusieurs reprises depuis 2007 aux travaux d'harmonisation du comité central des compétences et ont ainsi été témoins de l'avancement des travaux des autres conseils de compétence. Leur participation soutenue à ces rencontres assurait la liaison entre le comité central et le conseil de compétence en communication. Cette approche itérative s'est poursuivie jusqu'à ce qu'aucun nouveau contenu ne soit plus proposé par les membres du conseil de compétence et que ces derniers aient tous acquis la conviction que l'univers de la communication professionnelle en santé était couvert. De plus les travaux du conseil de compétence ont été formellement présentés aux membres du comité central des compétences de la faculté de médecine qui les ont révisés et commentés. Enfin, la validation élargie des contenus développés s'est aussi effectuée par la présentation des travaux du conseil de compétence en communication dans le cadre de divers congrès nationaux et internationaux ${ }^{[12-15]}$. Le groupe a ainsi tiré profit des apports de collègues de la francophonie et d'experts en communication médicale de la communauté scientifique internationale.

\section{Résultats}

\section{Définition de la compétence communicationnelle}

La communication professionnelle est une forme particulière de communication interpersonnelle. Dans le domaine médical, elle se définit comme un échange, verbal ou par tout autre moyen, entre un médecin, le patient, les proches de celui-ci, un autre professionnel de la santé ou avec un public. La communication professionnelle en médecine comporte essentiellement deux fonctions : 1) l'échange d'informations ; 2) le développement d'une relation.
Ainsi, la communication professionnelle en médecine implique la capacité à, d'une part, échanger de l'information et, d'autre part, établir une relation avec son interlocuteur en utilisant des moyens tels la parole, le geste, l'écriture ou des supports techniques comme le téléphone, le système de dictée, l'ordinateur, l'Internet, les courriels, etc.

La communication interpersonnelle réussie, dans une relation professionnelle, suppose une coopération entre les participants. Elle repose sur : 1) la maîtrise d'un répertoire de comportements communicationnels ; 2) la capacité d'adaptation aux différents interlocuteurs et aux contextes communicationnels et 3) la capacité d'atteindre ses buts professionnels et relationnels d'une manière compatible avec les buts et besoins du patient ou de tout autre interlocuteur rencontré dans l'exercice de ses fonctions ${ }^{[16]}$.

Une proposition de contenus et de processus organisés dans un outil didactique

L'exercice de réflexion entrepris par notre groupe s'inscrit dans la foulée des travaux de leaders contemporains reconnus dans le domaine de la communication médicale ${ }^{[2,8,9,11,16-18]}$ et s'appuie sur les données scientifiques disponibles en communication interpersonnelle et professionnelle. Ces données sont issues à la fois des sciences humaines (sociologie, psychologie, communication) et des sciences de la santé.

Nous rendons compte dans cet article des différents contenus et des processus associés à la communication en médecine, notamment de l'information véhiculée, de la relation, des contextes d'exercice et des circonstances particulières dans lesquelles cette communication médicale et professionnelle se déploie.

La représentation graphique qui nous semblait le mieux rendre compte de la complexité de cette compétence et des articulations entre ses diverses constituantes est celle d'un ensemble de cercles concentriques qui s'emboîtent les uns dans les autres. Cette représentation graphique (figure 1) 


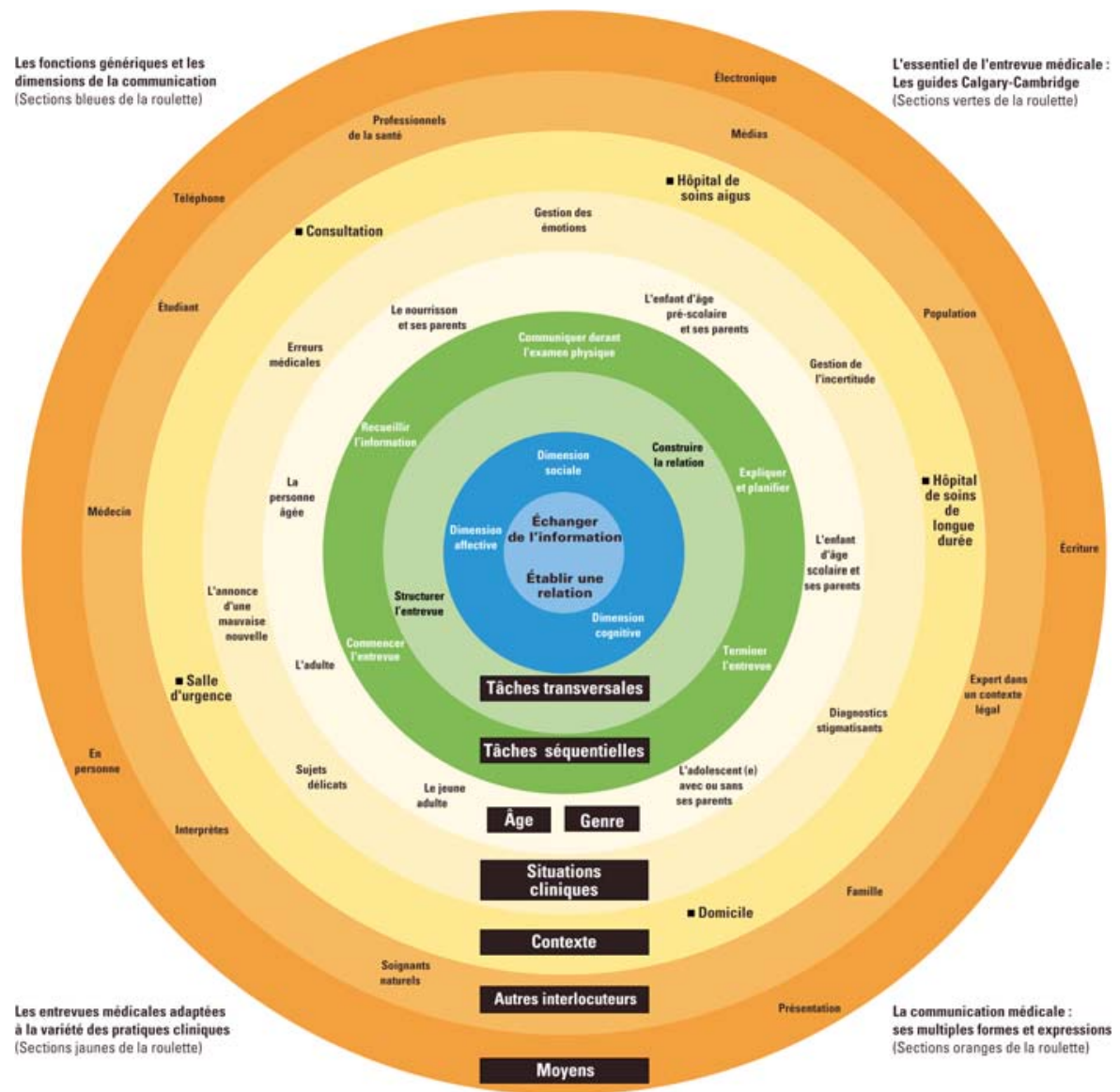

Fig. 1. Roulette combinatoire de la communication professionnelle. Inspirée des travaux de M. von Fragstein, J. Silverman, A. Cushing, S. Quilligan, H. Salisbury, C. Wiskin on behalf of the UK Council for Clinical Communication Skills Teaching in Undergraduate Medical Education (2008). NB : Les couleurs indiquées n'apparaissent en tant que telles que sur la version électronique de l'article; elles apparaissent sous forme de nuances de gris dans la version imprimée de l'article.

visait dès le départ à proposer un outil pratique pour les cliniciens et les enseignants. Nous l'avons intitulé «roulette», terme fréquemment utilisé au Québec pour décrire les petits documents plastifiés avec cercles rotatifs mobiles utilisés dans le cadre des activités cliniques, par exemple pour calculer la date prévue de l'accouchement, le moment des injections de contraceptifs à longue durée d'action ou le calendrier des vaccinations chez les jeunes enfants. Une telle «roulette » est aussi disponible pour décrire les difficultés d'apprentissage des étudiants en médecine ${ }^{[19]}$. Ce choix graphique et conceptuel est largement inspiré du modèle proposé par le «UK council for clinical communication skills teaching in undergraduate medical education » et publié récemment par von Fragstein et al. ${ }^{[7]}$. Toutefois, nous en avons modifié le contenu et son organisation en plaçant en son centre les fonctions et dimensions génériques de toute communication. Nous l'avons qualifiée de «combinatoire » pour illustrer son caractère dynamique qui permet d'aligner les éléments d'un cercle avec ceux d'un autre offrant ainsi une myriade de combinaisons possibles entre les constituantes.

La « roulette combinatoire » comporte neuf cercles distincts, chacun représentant une constituante de la communication. Ces constituantes sont regroupées en quatre métacatégories. Le tableau II présente l'ensemble des métacatégories et leurs constituantes. Le principe général d'organisation des 
Tableau II. Les neuf constituantes de la communication regroupées en quatre métacatégories.

\begin{tabular}{|c|c|c|c|}
\hline Métacatégorie & Titre & $\begin{array}{l}\text { Cercles } \\
\text { correspondan } 1\end{array}$ & Constituantes \\
\hline \multirow[t]{2}{*}{$\begin{array}{l}1 \\
\text { (Bleu) }\end{array}$} & \multirow[t]{2}{*}{$\begin{array}{l}\text { Les fonctions génériques et les di- } \\
\text { mensions de la communication }\end{array}$} & 1. & $\begin{array}{l}\text { - Échanger information } \\
\text { - Établir une relation }\end{array}$ \\
\hline & & 2. & - Dimensions cognitive, affective et sociale \\
\hline \multirow[t]{2}{*}{$\begin{array}{l}2 \\
\text { (Vert) }\end{array}$} & \multirow[t]{2}{*}{$\begin{array}{l}\text { L'essentiel de l'entrevue médicale : } \\
\text { les guides Calgary-Cambridge }\end{array}$} & 3. & - Les tâches transversales \\
\hline & & 4. & - Les tâches séquentielles \\
\hline \multirow[t]{3}{*}{$\begin{array}{l}3 \\
\text { (Jaune) }\end{array}$} & \multirow[t]{3}{*}{$\begin{array}{l}\text { Les entrevues médicales adaptées à } \\
\text { la variété des pratiques cliniques }\end{array}$} & 5. & - Âge et genre \\
\hline & & 6. & - Situations cliniques \\
\hline & & 7. & - Contextes ou milieux de soins \\
\hline \multirow[t]{2}{*}{$\begin{array}{l}4 \\
\text { (Orangé) }\end{array}$} & \multirow[t]{2}{*}{$\begin{array}{l}\text { La communication médicale : ses } \\
\text { multiples formes et expressions }\end{array}$} & 8. & - Autres interlocuteurs \\
\hline & & 9. & - Moyens \\
\hline
\end{tabular}

NB : Les couleurs indiquées n'apparaissent en tant que telles que sur la version électronique de l'article; elles apparaissent sous forme de nuances de gris dans la version imprimée de l'article.

contenus dans cette représentation est de placer en son centre les concepts les plus généraux et abstraits et d'évoluer vers les concepts les plus spécifiques et concrets en périphérie. Ainsi, les deux fonctions qui définissent toute communication, c'est-à-dire échanger de l'information et entrer en relation, sont placées au centre de la figure, alors que d'autres contenus, tels que «se servir du courriel pour communiquer », se retrouvent vers l'extérieur de la roulette (figure 1).

\section{La première métacatégorie : les fonctions génériques et les dimensions de la communication (cercles 1 et 2)}

Cette première métacatégorie définit les deux fonctions inhérentes à toute communication (cercle 1) et identifie les trois dimensions (cercle 2) à prendre en compte lors de ces communications.

Cercle 1 : Dans ce premier cercle, nous incluons les deux fonctions de la communication : échanger de l'information et développer une relation.

En communication professionnelle médicale, ces deux fonctions sont nécessaires aussi bien dans les communications que les médecins entretiennent avec les patients que dans celles qu'ils entretiennent avec l'ensemble des individus qu'ils rencontrent dans l'exercice de leurs fonctions. Ces deux fonctions sont centrales, selon nous, dans le cadre de l'exercice de la médecine et constituent en fait les deux capacités que les médecins doivent maîtriser. La capacité à échanger de l'information de façon efficace doit être placée au centre de la formation médicale étant donné la nature du travail à effectuer, par exemple poser un diagnostic, informer le patient ou une équipe de soins et suggérer un traitement. Cet échange d'information se fait dans le cadre d'une relation qui modulera l'ensemble des échanges du professionnel. Les informations échangées et les relations développées le seront avec plusieurs types de personnes, le patient d'abord mais aussi ses proches, l'ensemble des collègues et des autres membres de l'équipe de soins.

\section{1 Échanger de l'information}

1.1.1 Échanger efficacement de l'information avec les patients et leurs proches, se manifeste par la capacité de :

- comprendre leur expression verbale et paralinguistique ; 
- comprendre leur langage non verbal ;

- utiliser une information valide d'un point de vue scientifique, basée sur les données probantes, pour soutenir les opinions médicales ;

- identifier les contenus à présenter en tenant compte du contexte de soins, de la nature du problème de santé, de leurs caractéristiques individuelles et de leurs besoins ;

- adapter l'information à leur niveau de littératie, en particulier au sujet du diagnostic, du traitement et du pronostic ${ }^{[20]}$;

- adapter l'information au médium de communication utilisé (la parole, l'écrit, le téléphone ou la communication électronique);

- vérifier leur compréhension de l'information transmise;

- faire la distinction entre :

- informer

- expliquer (ex. à quoi attribuer la maladie)

- user de raisonnements et d'arguments dans le dialogue

- analyser les raisonnements ou arguments de l'interlocuteur pour y répondre et développer de nouveaux raisonnements plus conformes à la réalité médicale.

1.1.2 En plus des capacités mentionnées ci-dessus, l'échange efficace de l'information avec les collègues, les étudiants, les membres de l'équipe soignante ou du personnel de soutien se manifeste par la capacité de :

- identifier les contenus à présenter en tenant compte du rôle et du statut de l'interlocuteur ;

- tenir compte du contexte dans lequel la communication se produit (en public/en privé).

\section{2. Établir une relation}

Les relations que le médecin développe dans l'exercice de ses fonctions présentent des particularités. Il faut bien mettre en évidence les limites des relations professionnelles dans le temps, dans l'espace et dans les objets de discussion reconnus comme pertinents dans ce type de relation, ainsi que le rôle particulier qu'un médecin doit jouer dans le cadre de ce type de relation. De surcroît, il est important de comprendre la nature thérapeutique de la relation entretenue avec un patient et ses proches et en particulier son caractère asymétrique. Le médecin possède une compétence médicale mais le patient est la personne possédant une expérience de la maladie et de ses symptômes ${ }^{[21,22]}$.

1.2.1 Établir une relation avec les patients et leurs proches dans un cadre thérapeutique se manifeste par la capacité de :

- faire preuve de courtoisie, de respect, d'honnêteté et de sensibilité, c'est-à-dire de traiter les patients et leurs proches avec égards et considération par la parole et par les gestes ;

- préserver leur dignité, leur amour propre, leur fierté ou leur honneur ;

- établir et maintenir une relation chaleureuse, accueillante dans le respect de son rôle professionnel, des prescriptions morales, sociales, religieuses attendues par la personne dans les limites de ce qui est socialement et médicalement admis ;

- établir et maintenir une relation fondée sur la confiance, l'éthique et le respect, notamment des convictions et croyances des patients et de leurs proches (sauf les exceptions prévues par le code de déontologie et la loi);

- établir et maintenir une relation où le professionnel guide le patient dans sa démarche thérapeutique (exercice d'un leadership) ;

- exercer une influence (convaincre) sur le patient concernant les questions médicales ;

- gérer les potentiels conflits interpersonnels et les désaccords, par exemple sur les définitions du problème, les objectifs, le choix et les modalités de traitement et les rôles de chacun ${ }^{[23]}$.

1.2.2 Établir une relation dans le cadre d'échanges avec des collègues, des étudiants, des membres de l'équipe soignante ou du personnel de soutien, se manifeste par la capacité de :

- faire preuve de courtoisie, de respect, d'honnêteté, c'est-à-dire traiter leurs divers interlocuteurs avec égards, considération et respect par la parole et par les gestes ; 
- établir et maintenir des relations de travail professionnelles en tout temps respectueuses de la dignité de leur interlocuteur et appropriées au rôle attendu de chacun dans le contexte.

Cercle 2 : Dans ce deuxième cercle, nous incluons les dimensions sociale, cognitive et affective dont le médecin doit tenir compte lorsqu'il gère les aspects interactifs des échanges avec ses interlocuteurs au cours de l'exercice de ses fonctions professionnelles.

2.1 Sociale : Être capable d'adapter ses propos en fonction des caractéristiques sociales de la personne, comme sa religion, son appartenance culturelle, son statut économique, etc.

2.2 Cognitive : Être capable d'adapter ses propos au niveau de fonctionnement cognitif de la personne, de ses représentations, de son niveau d'éducation et de littératie en matière de santé.

2.3 Affective : Être capable d'identifier les sentiments et les émotions de son interlocuteur et d'exprimer cette compréhension sans blesser, heurter ou gêner d'une quelconque manière. Par exemple, il doit être capable de ressentir et d'exprimer, selon les circonstances, de la sympathie, de la compassion et de l'empathie.

\section{La deuxième métacatégorie : L'essentiel de l'entrevue médicale, les guides Calgary-Cambridge (cercles 3 et 4)}

La faculté de médecine de l'Université de Montréal a, il y a bientôt dix ans, adopté l'approche CalgaryCambridge ${ }^{[8,17]}$ pour l'enseignement de l'entrevue médicale, parce qu'elle intègre les principes du modèle de la relation centrée sur le patient et qu'elle s'appuie sur des données probantes tant dans le domaine de la santé que de l'éducation.

Cette deuxième métacatégorie présente donc l'entrevue médicale selon le modèle CalgaryCambridge ainsi que les pré-requis nécessaires à sa mise en œuvre. Les tâches transversales de l'entrevue (cercle 3) et les tâches séquentielles de l'entrevue (cercle 4) y sont proposées.

La maîtrise des habiletés communicationnelles fondamentales énumérées ci-dessous s'avère un pré-requis pour la mise en œuvre de l'entrevue médicale. Voici quelques exemples d'habiletés communicationnelles essentielles à la réalisation d'un entretien avec un patient ou ses proches :

- poser des questions ouvertes ou fermées de façon judicieuse ;

- écouter attentivement;

- interrompre avec tact l'interlocuteur si nécessaire ;

- identifier les indices verbaux et non verbaux émis par l'interlocuteur et y répondre;

- faire des résumés périodiquement;

- annoncer les transitions ;

- utiliser le reflet et l'empathie de façon judicieuse ;

- vérifier sa propre compréhension des faits, des représentations et des perceptions rapportées ;

- vérifier la compréhension du patient ou de ses proches au sujet du problème, de l'investigation ou du traitement.

De plus, le médecin doit démontrer une capacité à :

1- Lever les obstacles à la communication, que ceuxci relèvent de :

- la personne (déficit de la vue, de l'ouïe, de la parole, réduction de la mobilité)

- l'environnement (bruit, organisation spatiale);

- la situation clinique (douleur aiguë, fatigue, nausée, etc.).

2- Maîtriser la langue d'usage de son interlocuteur et employer le paralinguistique et le non verbal de façon culturellement appropriée.

Cercle 3 : Les tâches transversales : structurer l'entrevue et construire la relation. Nous renvoyons le lecteur aux références suivantes pour une description détaillée du contenu des Guides CalgaryCambridge ${ }^{[2,24]}$. Les tâches transversales sont celles qui ont cours tout le long de l'entrevue.

3.1 Structurer l'entrevue constitue la capacité à organiser le déroulement des échanges dans une structure logique et fluide, dans le respect des aspects affectifs et relationnels des échanges. 
3.2 Construire la relation reprend les capacités présentées dans la première métacatégorie mais les auteurs insistent ici sur les moyens mis en œuvre pour y arriver. (Voir cercle 7 pour une typologie des relations.)

Cercle 4 : Dans ce cercle, nous retrouvons les tâches séquentielles, c'est-à-dire les étapes de l'entrevue qui se déroulent habituellement dans l'ordre suivant :

4.1 Commencer l'entrevue.

4.2 Recueillir l'information.

4.3 Communiquer durant l'examen physique.

4.4 Expliquer et planifier.

- Expliquer le diagnostic ;

- Expliquer l'investigation;

- Expliquer le plan de traitement;

- Non pharmacologique (i.e. les habitudes de vie);

- Pharmacologique;

- Planifier le suivi.

4.5 Terminer l'entrevue.

La troisième métacatégorie : les entrevues médicales adaptées à la variété des pratiques cliniques (cercles 5, 6 et 7).

Cette métacatégorie présente des familles de situation auxquelles le médecin sera exposé au cours de sa pratique et qui nécessiteront des adaptations de l'entrevue médicale "générique ». Ces familles de situations sont reliées au genre des patients, aux différents groupes d'âge (cercle 5), à des situations cliniques spécifiques auxquelles il pourrait être confronté (cercle 6) de même qu'à divers contextes de pratique (cercle 7 ).

Cercle 5 : Dans ce cinquième cercle, nous présentons le genre et les différents groupes d'âge que le médecin est amené à rencontrer dans sa pratique.

La communication informationnelle et relationnelle sera influencée par l'âge de(s) l'interlocuteur(s) et leur genre. Au cours de sa formation et tout au long de sa carrière, le médecin pourra être exposé à des patients de plusieurs groupes d'âge :

- Le nourrisson et ses parents ou tuteurs ;
- L'enfant d'âge pré-scolaire et ses parents ou tuteurs ;

- L'enfant d'âge scolaire et ses parents ou tuteurs ;

- L'adolescent(e) avec ou sans ses parents ou tuteurs ou amis ;

- Le jeune adulte, seul ou accompagné ;

- L'adulte, seul ou accompagné ;

- La personne âgée seule ou en présence de personnes accompagnatrices.

Le médecin devra moduler sa communication en fonction du stade de développement cognitif, affectif et relationnel associé à ces différentes périodes du cycle de la vie ${ }^{[25]}$. Un âge particulièrement délicat dans la dimension relationnelle de la communication entre le médecin et le patient demeure l'adolescence. C'est plus spécialement pendant l'adolescence et le début de l'âge adulte que la différence de genre peut le plus influencer la communication ${ }^{[26]}$. Les gestes et la communication pendant l'examen physique devront s'adapter également au genre du patient dans le respect de sa pudeur et de son intimité.

Cercle 6 : Nous regroupons ici un ensemble de situations cliniques qui comportent des enjeux spécifiques qui en augmentent la complexité par rapport aux situations cliniques habituelles. Toutes ces situations exigent le déploiement par le médecin d'habiletés communicationnelles sophistiquées.

Le médecin devra être capable de :

6.1 Voici une liste partielle des principales situations cliniques spécifiques que nous avons identifiées qui exigeront une adaptation de l'entrevue :

- problèmes de santé mentale (état psychotique, risque suicidaire et homicide, trouble de l'humeur, trouble anxieux, trouble de personnalité, etc.);

- problèmes de dépendance (alcoolisme, toxicomanie, etc.) ;

- problèmes stigmatisants (VIH, trouble de la personnalité, etc.) ;

- problèmes cognitifs (démence, déficit intellectuel, etc.);

- problèmes en lien avec la pauvreté ; 
- problèmes associés à un faible niveau de littératie ou d'éducation ;

- problèmes de violence et d'abus physique ou psychologique (agresseur et victime) ;

- problèmes d'origine génétique (risque et pronostic).

6.2 Voici une liste partielle des principales thématiques particulières que nous avons identifiées qui pourraient faire l'objet de discussions à un ou à divers moments de l'entrevue :

- incertitude des données scientifiques ;

- émotions et affects ressentis, agis, identifiés et à prévoir ;

- demandes inappropriées ;

- annonce d'une mauvaise nouvelle;

- divers sujets délicats (ex. pratiques sexuelles, orientation sexuelle, abus d'alcool, de médicaments prescrits ou de substances illicites);

- niveau de soins et mandat d'inaptitude ;

- difficultés associées à des différences culturelles et de langue ;

- difficultés associées à la divulgation de complications et d'erreurs médicales.

- reconnaître les enjeux communicationnels spécifiques à chacune de ces situations thématiques ;

- identifier les habiletés communicationnelles pertinentes pour aborder ces situations thématiques ;

- démontrer une capacité à utiliser et à adapter de manière souple et fluide les habiletés communicationnelles pertinentes pour atteindre les objectifs cliniques spécifiques à chaque situation thématique.

En particulier, le médecin devra être capable de :

- expliquer dans un langage simple et compréhensible pour le patient des données abstraites (vulgariser) ;

- expliquer sans faire peur ni détruire l'espoir (risques et pronostics) ;

- gérer le malaise (voire l'angoisse) du patient généré par l'absence de certitude sur la nature et l'évolution de son état ou l'efficacité du traitement.

Cercle 7 : Dans ce septième cercle, nous énumérons différents contextes de pratique et nous proposons une typologie de relations susceptibles de s'y développer.

7.1 Le contexte de pratique modifie l'échange d'informations et la relation que le médecin peut entretenir avec son patient, ses collègues et collaborateurs. Les principaux lieux identifiés sont :

- la salle d'urgence ;

- le cabinet de consultation ;

- l'hôpital de soins aigus ;

- l'hôpital de soins de longue durée ;

- le domicile du patient.

Ces divers contextes de pratique favorisent, à notre avis, certains types de relations et de communication. Il ne s'agit pas d'une association exclusive mais bien d'une correspondance entre le lieu, le rôle du médecin et la nature des problèmes qui y sont habituellement rencontrés.

7.2 Le conseil de compétence en communication de l'Université de Montréal reprend ici la typologie des relations médecin-patient proposée par Lussier et Richard ${ }^{[9]}$ pour décrire les diverses relations qui peuvent s'établir entre un médecin et un patient. Cette typologie repose sur deux caractéristiques cliniques des problèmes présentés par le patient, respectivement sa chronicité (aiguë/chronique) et sa gravité (bénin/grave). Il est essentiel que le médecin développe une sensibilité au contexte et aux rôles distincts qu'il est appelé à jouer pour être en mesure d'adapter sa communication.

- Expertise-prise en charge

- Expertise-guidance

- Partenariat

- Facilitation.

Chacun des types de relation énumérés ci-haut sera rapidement décrit sous l'angle du rôle que joue le médecin dans la relation et l'impact de l'adoption d'un tel rôle sur la nature de la communication entre les deux interlocuteurs. Pour une discussion plus 
élaborée de cette typologie le lecteur est invité à se reporter au texte original ${ }^{[9]}$.

\subsubsection{Expertise-prise en charge}

Rôle :

Dans les situations cliniques très aiguës où la vie du patient est en danger, il est attendu que le médecin prenne des décisions selon les règles de l'art, quitte à expliquer au patient a posteriori la nature des actions posées et les raisons de les avoir posées. Dans ce cas, l'expression de l'attention portée au bien-être du patient s'exprime par la mobilisation des ressources médicales pour traiter le patient.

\section{Communication :}

L'expression verbale est succincte et parfois limitée à des énoncés comme «Nous allons prendre soin de vous ». Elle s'ajoute au toucher de la main ou de l'avant-bras du patient. On qualifie la relation comme fortement asymétrique dans ce contexte de soin.

\subsubsection{Expertise-guidance}

Rôle :

Dans ce cas, l'urgence médicale cède la place à une situation clinique qui demande une intervention relevant de l'expertise médicale. Des situations cliniques, sans être urgentes, telles qu'une maladie chronique non maîtrisée ou des problèmes médicaux plus aigus (infections bénignes, traumatismes ou blessures mineures), exigent du médecin qu'il partage son opinion professionnelle avec le patient. Il est aussi possible de discuter du traitement avec le patient et de considérer un certain nombre d'alternatives.

\section{Communication :}

L'objectif principal est d'informer le patient et de s'assurer de sa compréhension du problème et de son traitement. Dans ce rôle, le médecin peut introduire des aspects psychosociaux et tenir compte explicitement des opinions et croyances du patient. La relation est toujours asymétrique mais elle laisse plus de place à la collaboration entre le médecin et le patient.

\subsubsection{Partenaire}

Rôle :

Les situations cliniques auxquelles nous nous référons ici sont les maladies chroniques stables, telles que, par exemple, le diabète, l'hypertension, la dyslipidémie, l'arthrose ou l'ostéoporose. Dans ce contexte, le médecin est appelé à soutenir le patient dans son processus d'auto-soins et à vérifier l'atteinte des cibles de soins fixées de façon collaborative.

Communication :

Dans le cas du suivi au long cours des maladies chroniques, les connaissances du patient au sujet de son problème et ses compétences pour le gérer adéquatement se développent graduellement. Ainsi, l'asymétrie initiale de la relation entre le médecin traitant et son patient s'atténue. L'objectif principal du médecin devient donc de maintenir la motivation du patient à maîtriser le problème de santé. Dans ce contexte, les discussions sur les dimensions psychosociales, les opinions et croyances du patient sont pertinentes car elles offrent un levier significatif pour aider le médecin à soutenir les actions du patient que ce soit dans le changement ou le maintien d'habitudes de vie ou de la prise d'un médicament.

\subsubsection{Facilitateur}

Rôle :

Dans le cas de maladies chroniques bien maîtrisées par le patient, le rôle principal du médecin est de s'assurer que la maladie demeure contrôlée. Communication :

Dans le contexte du suivi des maladies chroniques telles que le colon irritable, le reflux gastroœsophagien, le diabète, l'asthme, la maladie pulmonaire obstructive chronique, le patient peut avoir développé une bonne compréhension de sa maladie et être capable d'évaluer l'évolution de son état et d'y réagir de manière appropriée. Dans ces circonstances, l'objectif du médecin est de maintenir la motivation du patient mais aussi de participer à l'augmentation ou au raffinement de ses connaissances et de répondre aux différentes demandes 
Le problème, le contexte de soins et le type de relation

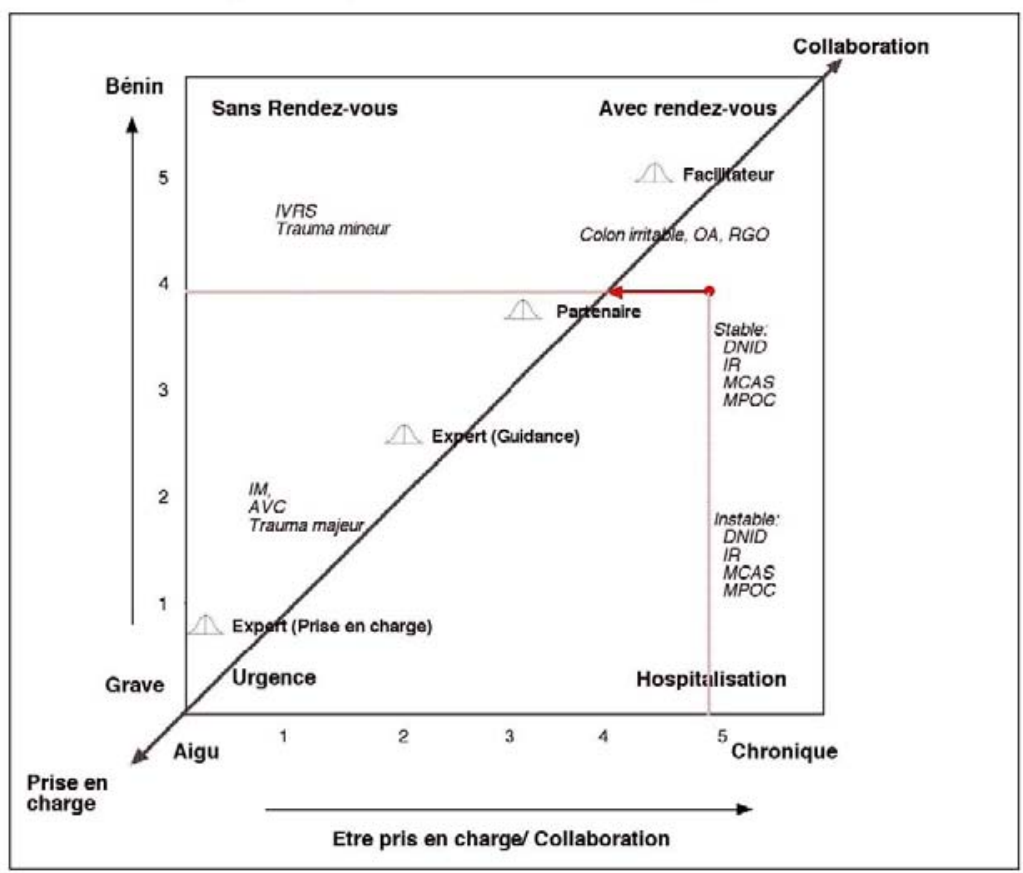

Fig. 2. Contexte de soins et types de relations. Figure extraite de Lussier MT, Richard C. (2008) ${ }^{[9]}$.

d'informations sur le traitement ou ses alternatives. Dans ce contexte, les dimensions psychosociales et les habitudes de vie occupent une plus grande place et font partie des facteurs discutés pour l'implantation du traitement. Le patient est invité à donner son point de vue et un véritable dialogue peut s'engager sur le plan de traitement.

La figure 2 illustre comment, à partir de deux dimensions des problèmes cliniques présentés par le patient, respectivement sa chronicité (aiguë/chronique) et sa gravité (bénin/grave), il est possible de définir les types de rôles que les médecins risquent d'adopter ainsi que les divers contextes de pratique. La diagonale représente l'évolution d'une relation de type expert-prise en charge complètement asymétrique vers une relation de collaboration. Dans chaque situation, nous situons le comportement le plus probable, d'autres comportements sont également possibles, ce qui est illustré par la courbe normale associée à chacun des noms de relations. Une discussion approfondie de cette typologie et de sa représentation se trouve dans l'article original ${ }^{[9]}$.
La quatrième métacatégorie : La communication médicale : ses multiples formes et expressions (cercles 8 et 9)

Cette dernière métacatégorie a pour but de rendre compte d'une part, de la multiplicité des interlocuteurs avec lesquels le médecin est susceptible d'interagir dans le cadre de ses fonctions professionnelles (cercle 8) et d'autre part, de la variété des moyens de communication qu'il pourra utiliser pour échanger avec ses interlocuteurs (cercle 9). Traditionnellement, la littérature scientifique sur la communication médicale traite très peu des habiletés nécessaires pour communiquer efficacement dans ces circonstances.

Cercle 8 : Dans le huitième cercle, nous présentons les autres interlocuteurs, en lien ou non avec le patient, avec qui le médecin est amené à interagir. Les communications doivent être modulées en fonction :

- du lien hiérarchique ou d'autorité qui unit les interlocuteurs ; 
- des droits et responsabilités de chacun des interlocuteurs dans la relation;

- du degré de connaissance des interlocuteurs et du degré approprié de familiarité selon le contexte;

- du respect de la confidentialité des échanges, par exemple en étant en mesure d'identifier ce qui relève des domaines public ou privé.

8.1 Dans la sphère professionnelle, le médecin entrera en relation avec les « autres » interlocuteurs à titre de :

- collègue médecin ;

- enseignant auprès d'étudiants, de résidents, de collègues professeur ;

- collaborateur avec des professionnels de la santé autres que médecin;

- responsable hiérarchique ou employeur de personnel de soutien ;

- expert de contenu dans le domaine de la santé vis-à-vis des médias, de la population et des organismes dans la collectivité ;

- expert de contenu médical dans un contexte légal, au tribunal, en présence d'agents législatifs, de curateurs et de tuteurs.

8.2 Dans la sphère « soignante », le médecin entrera en relation avec les interlocuteurs suivants :

- membres de la famille, proches et soignants naturels ;

- interprètes, soit professionnels, soit membres de la famille.

Cercle 9 : Dans ce neuvième cercle, nous détaillons différents moyens utilisés par le médecin dans le cadre de ses fonctions.

\subsection{En présence}

9.1.1 La parole en présence de son interlocuteur. C'est la communication la plus riche et celle qui permet le plus facilement de s'ajuster aux réactions de l'interlocuteur.

9.1.2 Le téléphone. C'est une communication moins riche que la précédente puisque les informations visuelles sont perdues. Cependant, comme il y a beaucoup de redondance entre le visuel et le vocal, elle permet de conserver une grande qualité aux messages grâce au paralinguistique qui reste accessible (les pauses, les hésitations, les constructions de phrases). De plus, par le fait qu'elle se déroule en temps réel, cette forme d'échange permet facilement de s'ajuster aux réactions de l'interlocuteur et d'introduire des nuances dans les propos.

\subsection{Par voie électronique}

9.2.1 Les courriels et les messages textes offrent une communication pauvre qui peut induire des lectures erronées du message, par l'absence du non-verbal et du paralinguistique. Les corrections se font lentement (communication asynchrone) et il est difficile de rectifier les malentendus. Les locuteurs tentent de compenser en utilisant une iconographie (par exemple un «bonhomme sourire ») sans succès garanti et qui, de plus, risque d'être inapproprié dans le contexte d'un échange professionnel formel.

9.2.2 La communication par internet avec vidéo ou par visioconférence présente un médium riche puisqu'elle permet de retrouver l'image (non verbal) et la conversation en direct. La spontanéité des échanges est perdue avec de multiples personnes. Les coûts d'installation de cette technologie et la nécessité de la présence d'un technicien limitent, pour l'instant, son utilisation fréquente.

9.2.3 Le site web des cliniques et des hôpitaux offre un médium pauvre avec un échange d'information le plus souvent écrit et unidirectionnel. Confectionné souvent sur mesure, il possède une interface de qualité variable pour l'utilisateur usuel et les délais fréquents de correction des informations induisent des erreurs dans leur transmission.

\subsection{Par écrit}

9.3.1 Le dossier médical papier, comme le dossier électronique, constitue une communication très pauvre puisqu'il est unidirectionnel et ne sous-tend que les impressions a posteriori d'un seul des interlocuteurs. Il témoigne des interprétations et des conclusions du médecin selon sa propre organisation 
informationnelle et fait état du suivi entre les rencontres. Il est malheureusement trop souvent difficile à lire à cause de la pauvre calligraphie ${ }^{[27,28]}$.

9.3.2 La rédaction d'une demande de consultation ou d'examen exige de synthétiser les informations cliniques essentielles qui pourront guider l'analyse et l'interprétation des données recueillies par l'autre professionnel. Les informations transmises devraient inclure, minimalement, les éléments suivants : l'âge du patient, son genre, les diagnostics connus et pertinents par rapport à la demande, la raison précise de la demande de consultation (problème de diagnostic, d'investigation ou de traitement) ou de la demande de l'examen paraclinique (ce que l'on cherche à identifier ou éliminer) et l'identité du requérant et ses coordonnées. Le style doit être clair et concis, le ton neutre.

9.3.3 La rédaction d'un rapport écrit de consultation doit inclure l'identité du médecin requérant et la raison de la demande. Il doit aussi inclure l'identification du patient (nom, date de naissance, numéro d'assurance maladie et numéro de dossier). Il est habituellement construit selon les rubriques traditionnelles de toute "histoire de cas/anamnèse ", à savoir la raison de la demande, l'histoire de la maladie ou du problème actuel incluant les résultats des investigations et des autres consultations qui ont été effectuées, la revue des systèmes, les antécédents médicaux et chirurgicaux, personnels et familiaux, pertinents, les habitudes de vie pertinentes, la liste des médicaments et les allergies, les observations de l'examen physique, les résultats des examens paracliniques effectués par le spécialiste (par exemple : gastroscopie, bronchoscopie, biopsie, etc.), le diagnostic et les propositions de traitement et de suivi. Le médecin doit signer et dater son rapport.

La forme de ces rapports est très standardisée. Le ton de ce rapport doit être formel et neutre. Par rapport à la discussion de cas en présence du médecin demandeur et du médecin consultant, la quantité d'information est plus restreinte et il y a moins de place pour la discussion de zones grises.

9.3.4 La rédaction d'un rapport écrit d'examen paraclinique ou de protocole opératoire doit contenir l'identification du patient (nom, date de naissance, numéro d'assurance maladie et numéro de dossier). Il doit préciser la nature de l'examen ou de la chirurgie effectuée et les conditions dans lesquelles l'examen ou la chirurgie ont été effectuées. Il doit décrire les résultats pertinents selon la spécialité ou le type d'examen ou de procédure effectués et conclure quant au résultat de l'examen et la nécessité ou non d'effectuer des examens complémentaires. Le médecin doit signer et dater son rapport. Il doit s'assurer que le rapport soit acheminé au médecin requérant. La forme du rapport et ses limites, lorsqu'on les compare à une discussion de vive voix, sont les mêmes que pour la rédaction d'un rapport de consultation.

9.3.5 La dictée d'une lettre, d'un rapport ou d'un protocole opératoire doit être énoncée clairement de façon à diminuer les erreurs potentielles de transcription. Lorsqu'elle est transcrite, il est de la responsabilité du médecin de la relire pour s'assurer de l'exactitude de son contenu avant de la signer et de l'acheminer au destinataire.

9.3.6 La rédaction de rapports d'expertise, d'articles de vulgarisation ou d'articles scientifiques respectent des normes d'écriture qui leur sont propres et qui exigent du médecin une formation spécifique et une supervision qui n'est habituellement pas offerte dans le cadre des programmes de médecine ou de résidence. Le lecteur peut consulter les références proposées pour en connaître davantage ${ }^{[29-31]}$. Cette compétence communicationnelle spécifique ne fera pas ici l'objet d'autres commentaires pour cette raison.

\subsection{Présentation - conférence}

Les présentations à un groupe, que ce soit une présentation magistrale ou un atelier, exigent une maîtrise du contenu scientifique à partager et 
des habiletés communicationnelles pour favoriser la transmission des informations.

9.4.1 La présentation en plénière (conférence magistrale) représente un échange séquentiel dans lequel il y a une asymétrie franche entre les interlocuteurs, en l'occurence le présentateur actif et son auditoire réceptif. Il s'agit habituellement d'une présentation orale soutenue par du matériel visuel sous forme de texte allégé, de figures, de tableaux ou même de séquences audio-vidéo. Bien que souvent appréciée des auditoires, ce genre de communication n'est pas très efficace pour une rétention des informations par les auditeurs. Une période de questions à la fin de la présentation permet quelques échanges entre l'auditoire et le présentateur. Il existe des formations spécifiques pour améliorer la qualité des conférences magistrales mais ceci ne peut faire l'objet de cet article $^{[32]}$.

9.4.2 La présentation d'un atelier interactif exige du présentateur une attention particulière à l'organisation et à l'intégration des activités de travail en petits groupes. Le présentateur tient le rôle d'un animateur. Il encadre les échanges entre les participants ce qui exige une capacité d'écoute attentive, de reformulation et de synthèse des propos et des arguments de même que l'usage de questions ouvertes qui suscitent les échanges. Il doit gérer les interactions du groupe en présentant les règles de fonctionnement du groupe et en s'assurant de leur respect. Il est le gardien du temps et est responsable du déroulement harmonieux des échanges et de l'atteinte des objectifs.

Des formations à l'animation d'atelier et à la gestion des discussions en petits groupes sont disponibles $^{[33,34]}$.

\section{Roulette combinatoire : des exemples d'usage}

La communication ainsi découpée en quatre métacatégories organisatrices offre une carte qui permet, tant à l'enseignant qu'à l'apprenant, de s'orienter plus facilement dans les constituantes de cette compétence. Elle se prête bien à une introduction aux fonctions et aux dimensions de la communication professionnelle lors de l'enseignement théorique et clinique.

Dans sa version dynamique avec les cercles qui pivotent les uns par rapport aux autres, la roulette utilisée alors comme un système combinatoire, permet d'identifier avec précision et selon leur contexte, les constituantes et stratégies communicationnelles d'une entrevue. Ainsi, en préparation à une supervision clinique par observation directe, l'enseignant et l'apprenant peuvent identifier pour ensuite les travailler, les éléments de communication pertinents par rapport à l'entrevue à venir. La roulette peut également soutenir la rétroaction que l'enseignant donnera à l'apprenant, en l'aidant à préciser dans l'action, les éléments communicationnels d'intérêt lors de l'échange observé et permettre la discussion de stratégies communicationnelles alternatives.

Par ailleurs, la roulette devient un outil pour élaborer des vignettes cliniques. Il s'agit alors de choisir un élément par cercle en procédant des cercles internes vers les cercles externes. L'ensemble des éléments constitue les bases du cas clinique. Un ensemble de permutations fort impressionnant vient ainsi soutenir l'enseignant en mal de cas concrets. Par exemple, dans une formation à la communication, l'enseignant pourrait construire une vignette clinique dans un contexte où le temps est restreint (les urgences) et où un sujet « délicat» (possibilité d'abus physique) doit être abordé de façon claire et structurée en démontrant le niveau de sensibilité requis par la discussion d'une telle hypothèse diagnostique. Les choix sur la roulette seraient les suivants : Cercle 1 : Établir une relation;

Cercle 2 : Dimension affective ;

Cercle 3 : Structurer l'entrevue ;

Cercle 4 : Expliquer et planifier;

Cercle 5 : Femme, 50 ans ;

Cercle 6 : Victime d'abus physique ;

Cercle 7 : Consultation sans rendez-vous pour une douleur au bras ;

Cercle 8 : Seule;

Cercle 9 : En personne. 


\section{Discussion}

L'explicitation didactique proposée des constituantes de la communication professionnelle en médecine et de son organisation en un ensemble de cercles concentriques s'appuie sur une démarche systématique et itérative conduite au sein du conseil de compétence en communication de la faculté de médecine de l'Université de Montréal. Chacun des membres du conseil a contribué à la vision proposée par son expertise spécifique et sa connaissance des pratiques médicales qui relèvent de sa spécialité. Cette «mise à plat » n'est pas exhaustive mais sa validité de contenu est assurée par le large accord qu'elle a suscité parmi les membres du conseil. Chacun des membres s'est assuré de la pertinence de la description des activités communicationnelles pour son champ d'activités cliniques respectif (spécialités médicales et chirurgicales). De plus, la validité apparente de la représentation proposée semble confirmée par les réactions très favorables des divers publics à qui la roulette a été présentée au cours des deux dernières années ${ }^{[12-15]}$

Cette représentation ne constitue pas encore un véritable modèle de la communication médicale. Cependant, la manière de décrire et de représenter l'ensemble des constituantes de la communication médicale n'est pas exempte de théorie. En effet, tout ordonnancement de la réalité repose implicitement ou explicitement sur une conception à propos de la nature de cette réalité. Ce découpage, qui ne fait pas partie de la réalité elle-même, induit, de par le choix des unités significatives proposées, une vision particulière de la communication et de la relation médecin-patient. Chaque découpage a des avantages mais comporte aussi des limites. Par exemple, le fait de suggérer l'existence d'un continuum relationnel qui privilégie tantôt le principe éthique de la bienfaisance et tantôt le principe éthique de l'autonomie permet de voir la réalité d'une certaine manière. Si nous acceptons l'existence d'un tel continuum relationnel, nous pouvons ensuite discuter l'intérêt, dans une situation clinique donnée (par exemple un patient se présentant avec douleur rétrosternale sévère par opposition à un patient consultant pour une recrudescence des symptômes d'un intestin irritable), d'adopter un rôle dans ce continuum relationnel qui soit le mieux adapté à cette situation clinique et au contexte de soins. Ce découpage qui propose l'existence de multiples relations possibles entre un médecin et son patient s'oppose à un modèle « universel » de la relation médecin-patient applicable dans toutes les situations. Ce répertoire de relations possibles suggère une grande souplesse dans l'adoption d'un ensemble de comportements communicationnels mais exige du clinicien une évaluation singulière et spécifique plutôt qu'une application mécanique et exclusive d'une façon de faire.

Nous avons placé, au centre de notre représentation, la théorie (connaissances), de laquelle découle l'action dans l'entrevue médicale puis dans les autres interactions professionnelles. Par ce choix nous annonçons une conception de l'approche à la communication qui suppose une préséance de la connaissance sur l'action qui, elle-même, se répartit entre ce qui est proximal à l'entrevue médicale et ce qui en est plus distal. Ce faisant, notre proposition a l'ambition de couvrir l'ensemble des activités de communication professionnelle auquel participe le médecin contemporain. Nous avons dû faire des choix dans une réalité multiple et complexe et, en conséquence, notre travail est donc à achever, à parfaire et à compléter.

De la même manière, nous avons fait le choix des guides Calgary-Cambridge pour décrire les habiletés communicationnelles utiles dans la conduite d'une entrevue médicale structurée et efficace. Ils ont été choisis parce que les habiletés communicationnelles qui y figurent ont toutes été identifiées comme étant associées à des meilleurs résultats de soins ${ }^{[8]}$. Il s'agit d'une approche qui ne se limite pas à un modèle théorique unique de la relation médecin-patient mais qui intègre l'ensemble des habiletés communicationnelles qui ont été démontrées efficaces. De plus, cette méthode est une des rares à présenter une approche intégrée et systématique de l'apprentissage de l'entrevue médicale ${ }^{[17]}$. Le conseil de compétence en communication était d'avis qu'il ne pouvait pas faire 
l'économie de l'usage d'une telle approche pour systématiser l'enseignement de l'entrevue médicale aux diverses étapes du curriculum des études médicales.

L'organisation de la représentation en une série de cercles concentriques fournit une vue d'ensemble des différentes constituantes de la communication professionnelle du médecin. La communication découpée en quatre métacatégories organisatrices offre pour ainsi dire une carte tant à l'enseignant qu'à l'apprenant, qui leur permet de s'orienter plus facilement dans ce territoire. Elle se prête bien à une introduction aux fonctions et aux dimensions de la communication professionnelle lors de l'enseignement théorique et clinique. Dans une version dynamique (avec les cercles qui pivotent les uns par rapport aux autres), la roulette devient un outil pour soutenir un enseignement précis et vivant.

Par ailleurs, les cliniciens enseignants à qui l'outil a été présenté ont reconnu l'utilité immédiate de cette roulette pour «nommer» avec une plus grande confiance ce qui a été fait et ce qui aurait pu être fait autrement du point de vue communicationnel. La roulette devient pour eux un outil puissant pour soutenir concrètement l'apprentissage de la communication, que ce soit lors de jeux de rôle ou de la supervision directe d'entrevues médicales.

\section{Conclusion}

Nos travaux constituent une proposition d'organisation des connaissances et des habiletés communicationnelles en médecine. Cette représentation ne constitue pas encore un véritable modèle. Il s'agit plutôt d'un proto-modèle de la communication médicale qui croyons-nous, fera partie intégrante d'un futur modèle.

Cette explicitation didactique est un préalable au développement de la compétence communicationnelle. Pour développer cette compétence, il faudra y ajouter une approche systématique et graduelle de la formation, au cours de laquelle la réussite de chacune des étapes repose sur leur maîtrise progressive. Ainsi, l'ensemble des formateurs disposera d'une identification des différentes constituantes d'une communication professionnelle en situation médicale et, bientôt, d'un outil pédagogique pour les aider à l'enseigner. Ce travail pourra également servir de base aux responsables pédagogiques facultaires pour organiser l'enseignement de la communication dans leur spécialité et selon le niveau de formation des étudiants.

\section{Contributions}

Claude Richard, Marie-Thérèse Lussier, Sophie Galarneau et Olivier Jamoulle ont contribué solidairement à l'écriture des versions successives du manuscrit.

\section{Références}

1. Millette B, Lussier MT, Goudreau J. L'apprentissage de la communication par les médecins : Une mission académique prioritaire. Pédagogie Médicale 2004;5:110-26.

2. Silverman J, Kurtz S, Draper J. Outils et stratégies pour communiquer avec le patient. Chêne-Bourg: Médecine \& Hygiène, 2010.

3. Giroux L. Les modèles de relation médecin-patient. In: Richard C, Lussier MT, éditeurs. La communication professionnelle en santé. Montréal: ERPI, 2005:113-43.

4. Beebe SA, Beebe SJ, Ivy DK. Communication: Principles for a lifetime (3th ed.). Boston (MA): Pearson Education, 2007.

5. Knapp M, Miller G. Handbook of interpersonal communication (2nd ed.). Thousand Oaks (CA): Sage, 1994.

6. Greene JO, Burleson BR. Handbook of communication and social interaction skills. Mahwah (NJ): Lawrence Erlbaum Associates, 2003.

7. von Fragstein M, Silverman J, Cushing A, Quilligan S, Salisbury H, Wiskin C. UK consensus statement on the content of communication curricula in undergraduate medical education. Med Educ 2008;42:1100-7.

8. Silverman J, Kurtz S, Draper J. Skills for communication with patients. 2nd ed. Abingdon (UK): Radcliffe, 2005.

9. Lussier MT, Richard C. En l'absence de panacée universelle. Can Fam Physician 2008;54:1096-9.

10. Frank JR. Le cadre de compétences CanMEDS 2005 pour les médecins. L'excellence des normes, des médecins et des soins. Ottawa: Le Collège royal des médecins et chirurgiens du Canada, 2005. 
11. Richard C, Lussier MT. La communication professionnelle en santé, ed. Montréal: ERPI; 2005.

12. Richard C, Lussier MT, Jamoulle O, Ayoub J, Dubois MJ, Galarneau $\mathrm{S}$ et al. La compétence en communication professionnelle : une suggestion de cursus longitudinal. $9^{e}$ Congrès national du Collège national des généralistes enseignants; 26-27 novembre 2009; Toulouse (France), 2009.

13. Richard C, Lussier MT, Jamoulle O, Galarneau S, Ayoub $\mathrm{J}$, Dubois MJ et al. La compétence en communication professionnelle : une suggestion de cursus longitudinal. $5^{\text {e }}$ Forum international francophone de pédagogie en sciences de la santé, 16-18 juin 2010 Québec (QC). In: Pédagogie Médicale 2010;11(Suppl. 1):S52.

14. Richard C, Lussier MT, Jamoulle O, Ayoub J, Dubois MJ, Galarneau $\mathrm{S}$ et al. Competence in professional communication: A developmental approach. Family Medicine FORUM 2010 du Collège des médecins de famille du Canada; 14-16 octobre 2010; Vancouver (BC) 2010.

15. Richard C, Lussier MT, Jamoulle O, Ayoub J, Dubois MJ, Galarneau S et al. Competence in professional communication: A developmental approach. EACH European Association of Communication in Healthcare; 5-8 septembre 2010; Verone, Italie 2010.

16. Street RLJ. Interpersonal communication skill in health care contexts. In: Greene JO, Burleson BR, éditeurs. Handbook of communication and social interaction skills. Mahwah (NJ): Lawrence Erlbaum Associates, 2003:909-34.

17. Kurtz S, Silverman J, Draper J. Teaching and learning communication skills in medicine (2nd ed.). Abingdon (UK): Radcliffe Medical Press, 2005.

18. Stewart M. Effective physician-patient communication and health outcomes: a review. Canad Med Assoc J 1995; 152:1423-33.

19. Lacasse M. Educational diagnosis and management of challenging learning situations in medical education. Québec (QC): Université Laval, 2009.

20. Richard C, Lussier MT. La littératie en santé, une compétence en mal de traitement. Pédagogie Médicale 2009;10:123-30.

21. McWhinney IR. Patient-centered and doctor-centered models of clinical decision making. In: Sheldom M, Brooke J, Rector A (Eds.). Decision making in general practice. New York (NY): Stockton Press, 1985:31-45.

22. Levenstein JH, McCracken EC, McWhinney IR, Stewart MA, Brown JB. The patient-centred clinical method.
A model for the doctor-patient interaction in family medicine. Fam Pract 1986;3:24-30.

23. Beaudouin C. Dire non à un patient: comment minimiser son insatisfaction tout en l'aidant. Le médecin du Québec 1999;34:35-42.

24. Richard C, Lussier MT, Kurtz S. Une présentation de l'approche Calgary-Cambridge. In: Richard C, Lussier MT, éditeurs. La communication professionnelle en santé. Montréal: ERPI 2005:291-324.

25. Marsh P, Gilmore R. Eye to eye. Topsfield: Salem House, 1991.

26. Girard M, Charbonneau L, Lambert Y, Richard C, Lussier MT. Les adolescents. In: Richard C, Lussier MT, éditeurs. La communication professionnelle en santé. Montréal: ERPI 2005:345-85.

27. Collège des médecins du Québec. La rédaction et la tenue des dossiers par le médecin en cabinet de consultation et en CLSC. Guide d'exercice du Collège des médecins du Québec. Montréal : Collège des médecins, 2006.

28. Collège des médecins du Québec. La tenue des dossiers par le médecin en centre hospitalier de soins généraux et spécialisés. Guide d'exercice du Collège des médecins du Québec. Montréal : Collège des médecins, 2005.

29. Bordage G. La préparation d'un article pour publication. Pédagogie Médicale 2002;3:237-48.

30. Bordage G, Dawson B. Planification d'une étude expérimentale et rédaction d'une demande de subvention en 8 étapes et 28 questions. Pédagogie Médicale 2003;4:314.

31. Bordage G, Guerin S. Le résumé structuré : un outil de lecture, d'évaluation et de rédaction. Pédagogie Médicale 2001;2:81-4.

32. Borduas F, Thivierge RL. L'expert-conférencier : Au-delà du rôle traditionnel et implications nouvelles pour la formation des formateurs. Pédagogie Médicale 2001;2:3741.

33. Girard G, Clavet D, Boulé R. Planification et animation de trois types d'ateliers pédagogiques. Pédagogie Médicale 2008;9:42-53.

34. Agora-Formation. Technique d'animation : le jeu de rôle. Fiche pratique 017. Pédagogie Médicale 2004;5:241-2.

Correspondance et offprints : Claude Richard, Équipe de recherche en soins de première ligne, Cité de la santé de Laval, Hôpital Cité-de-la-santé, Bureau DS-075, Centre de santé et de services sociaux de Laval, 1755, Bd René-Laennec, H7M 3L9 Laval, Québec, Canada.

Mailto : ell4400@videotron.ca 